\title{
Causality in Time-Neutral Cosmologies
}

\author{
Adrian Kent \\ Department of Applied Mathematics and Theoretical Physics, University of Cambridge, \\ Silver Street, Cambridge CB3 9EW, U.K.
}

(18 March 1997, revised 13 August 1998)

\begin{abstract}
Gell-Mann and Hartle (GMH) have recently considered time-neutral cosmological models in which the initial and final conditions are independently specified, and several authors have investigated experimental tests of such models.

We point out here that GMH time-neutral models can allow superluminal signalling, in the sense that it can be possible for observers in those cosmologies, by detecting and exploiting regularities in the final state, to construct devices which send and receive signals between space-like separated points. In suitable cosmologies, any single superluminal message can be transmitted with probability arbitrarily close to one by the use of redundant signals. However, the outcome probabilities of quantum measurements generally depend on precisely which past and future measurements take place. As the transmission of any signal relies on quantum measurements, its transmission probability is similarly context-dependent. As a result, the standard superluminal signalling paradoxes do not apply. Despite their unusual features, the models are internally consistent.

These results illustrate an interesting conceptual point. The standard view of Minkowski causality is not an absolutely indispensable part of the mathematical formalism of relativistic quantum theory. It is contingent on the empirical observation that naturally occurring ensembles can be naturally pre-selected but not post-selected.
\end{abstract}

\section{INTRODUCTION}

Standard quantum theory is applied in a time asymmetric way. The physical state of a quantum system is taken to be completely described by the state vector at any given time, which is taken to be derivable from past events. The state vector encodes all the available information about the future behaviour of the system, allowing probabilistic predictions about the results of any sequence of possible future measurements.

As Aharonov, Bergmann and Lebowitz (ABL) pointed out in their classic discussion 11 of time symmetry and quantum measurement, this time asymmetry derives not from the formalism of quantum theory but from the way we construct the statistical ensembles to which the theory is applied. If ensembles are constructed time symmetrically, using post-selection as well as pre-selection, then the probability distribution for the outcome of any series of experiments at intermediate times is time symmetric.

The familiar time asymmetry relies on the assumption that ensembles on which measurements have unambiguous probability distributions can be constructed via preselection alone. Every test to date supports this assumption, but it remains logically possible that it could be empirically refuted without necessarily invalidating the basic formalism of quantum theory.

Recently, Gell-Mann and Hartle (GMH) have proposed [6] a class of time-neutral cosmological models in which the initial and final quantum states are fixed independently. These models provide a foil against which to test the standard time asymmetric understanding of cosmol- ogy. We describe below a property of GMH time-neutral cosmologies which seems to have gone unnoticed, namely that it can be possible for observers in these cosmologies to send superluminal signals.

\section{TIME-NEUTRAL COSMOLOGIES: ASSUMPTIONS AND DEFINITIONS}

We consider, for simplicity, observers near one end of the cosmology, who experience a thermodynamic arrow of time that is well-defined and unidirectional throughout space during their era. We assume that the initial conditions are defined by some low entropy state, and adopt the observers' time conventions, referring to past and future, and to initial and final states, with respect to their thermodynamic arrow. We suppose that the observers can readily use present data to make inferences about past events, and can readily produce ensembles of quantum systems whose state is defined (or at least constrained) by a process of pre-selection.

If the final state is also of low entropy, there is no globally defined thermodynamic arrow of time. The final state need not be of low entropy, but it must, for the purposes of our argument, have some regularities. The observers must be able to detect and exploit features of the final state which allow them to identify ensembles of quantum systems that are pre-selected and post-selected in such a way as to alter the standard outcome probabilities predicted on the basis of pre-selection alone. As we will show, it is easy to construct examples of time-neutral 
cosmologies in which this necessary condition holds.

Any discussion of quantum theory in the context of cosmology goes outside the Copenhagen framework, and so raises difficult interpretational questions. GMH interpret their time-neutral cosmological models via the consistent histories formulation of quantum cosmology. [2] We focus here on the observational implications for standard quasiclassical observers (such as, hypothetically, ourselves) in a time-neutral cosmology, which allows us to leave aside most interpretational questions. Instead of considering all the infinitely many incompatible consistent sets defined by GMH, we suppose, as part of the definition of a time-neutral cosmological model, that one particular quasiclassical consistent set $\mathcal{S}$ has been fixed. All statements about quasiclassical events - the actions of observers, their manipulations of their experimental apparatus, and their observations - are statements which should in principle be understood in terms of quasiclassical variables defined by that fixed set. ${ }^{1}$

So, the full cosmological theory is defined by initial and final states, $\rho_{i}$ and $\rho_{f}$, which are non-orthogonal positive semi-definite matrices, and the specified quasiclassical consistent set of histories $\mathcal{S}$. We implicitly require also some theory of the hamiltonian and specification of canonical variables which allows us to make physical sense of the events abstractly specified in $\mathcal{S}$ allowing us, for example, to interpret suitable projections $P$ on Hilbert space in terms of local number density operators integrated over small regions, and so to interpret the statement that $P$ is realised as the statement that the density $d$ of matter in a certain specified volume lies in the range $d_{\min } \leq d \leq d_{\max }$.

The probabilities for particular quasiclassical events for example, a given observer pressing the switch of a signalling device - are now, in principle, calculable from the boundary conditions by the standard decoherence functional probability formula. [2]

A comment on free will is necessary at this point. When we press a switch, it generally seems to us that we could have chosen not to. ${ }^{2}$ If our pressing a switch is reliably correlated with someone else receiving a message - in such a way that we can give a direct physical explanation of the fact that the message will be received if and only if we press the switch - then we describe ourselves as sending a signal by the action of pressing the switch. This is the operational definition used in this paper when, for example, we speak about an observer choosing to send

\footnotetext{
${ }^{1}$ We assume here familiarity with the consistent histories approach to quantum cosmology. For reviews, attempts at a definition of quasiclassicality, and discussions of the problems see, for example, Refs. 1275 .

${ }^{2}$ Whether or not this impression of free choice is justifiable is, of course, beyond the scope of this paper!
}

a signal. We will show that time-neutral cosmological models in which the final state has a detectable regularity supply a mechanism by which intelligent observers can construct devices which allow them - in this operational sense - to send superluminal signals.

One can imagine pathological cosmological models in which, via some conspiracy of the boundary conditions, it is pre-determined that observer A will press a switch a number of times, and that observer $\mathrm{B}$ will receive a message at correlated times, although there is no direct physical link between the switch and the message. Such coincidences have no simple physical explanation: they can only be understood from a detailed analysis of the boundary conditions. It cannot then be said that pressing the switch causes the message to be received, in the conventional sense. As will be seen, pathologies of this sort are not invoked here.

We suppose that the specific quasiclassical events to be discussed occur in a small, flat region of space-time in roughly our own cosmological era - near the earth, in the near future, for example. We suppose too that (as is generally believed) the simplest non-relativistic version of the consistent histories formalism adequately describes localised quasiclassical physics: i.e., we take physical events to be defined by projections at given times in some fixed frame,

That is, we assume that some partial and coarsegrained quasiclassical history $H$, built from basic events in $\mathcal{S}$, and sufficient to describe the evolution of the classical structure of the universe up to the present, has already been realised. All subsequent probabilities should now, in principle, be calculated by conditioning on the realisation of $H$. To avoid the need for this, we consider events defined by interactions of pre- and post-selected quantum subsystems with classical measuring devices, and assume that the subsystems are uncorrelated with the quasiclassical degrees of freedom at all times between the initial condition and their first measurement, and again between their final measurement and the final condition.

\section{SUPERLUMINAL SIGNALLING}

A single example suffices to show that time-neutral cosmologies are not immune from superluminal signalling. It is hard to make the example very realistic, for several 
reasons. First, neither quantum cosmology nor quantum gravity are well understood. Second, there is no precise mathematical definition of quasiclassicality. Third, no detailed proposals for plausible time-neutral cosmologies have been made. Indeed, while time-neutral quantum theory itself is certainly consistent, it is less clear that time-neutral cosmological models with the specific properties that GMH consider [6] are consistent with present theories and observations. ${ }^{3}$ We can, however, produce an example of superluminal signalling under assumptions which, while contrived, are not excluded by any theoretical principle.

Suppose that the cosmology can be divided into two subsystems, which interact only briefly. The first subsystem includes an entire quasiclassical realm, containing observers, their measuring devices, and all the large-scale cosmological structure. It is to be described by some consistent set $\mathcal{S}$, which is sufficiently detailed to describe the actions of the observers and the experiments they carry out. We can assume that this realm, the observers and their experimental devices are very like our own, us and ours.

The second subsystem is a large supply of pairs of initially entangled particles initially in a singlet spin state

$$
|B\rangle=1 / \sqrt{2}\left(|\uparrow\rangle_{L}|\downarrow\rangle_{R}-|\downarrow\rangle_{L}|\uparrow\rangle_{R}\right)
$$

where the states $|\uparrow\rangle$ and $|\downarrow\rangle$ are the $\pm \frac{1}{2}$ eigenstates of $\sigma_{z}$, for a choice of the $z$ axis which is unambiguously defined by parallel transport throughout their propagation. These pairs are otherwise initially uncorrelated with each other and with the rest of the quasiclassical realm.

The pairs of particles propagate freely from the beginning of the cosmology, undergoing no significant interactions, with the $\mathrm{L}$ and $\mathrm{R}$ particles propagating in distinct directions, until they arrive at two regions populated by experimentalists. The experimentalists have set up measuring devices, which register the arrival of each particle and measure its spin, about some axis or other — the axes are initially altered quite often by the experimentalists, as they attempt to investigate the ensemble statistics for various choices of axes. Their measurements are idealised von Neumann measurements, so that the standard ABL time-neutral probability formula applies.

The particles arrive sequentially pair by pair: i.e the first particles to arrive at each apparatus are entangled, as are the second and every successive pair. These arrival events have a large spacelike separation. After a single measurement, they propagate freely off into empty space, never again undergoing any significant interaction until the end of the cosmology. At this point, they satisfy the

\footnotetext{
${ }^{3} \mathrm{~A}$ thoughtful discussion of some of the problems that arise in defining GMH time-neutral cosmologies can be found in Ref. [7].
}

final condition that the $\mathrm{R}$ particles are found, with certainty, in the state $|\uparrow\rangle_{R}$; the final state of the $\mathrm{L}$ particles is not constrained.

The full initial state for the cosmology is thus $\rho_{i} \otimes$ $\rho_{B} \otimes \cdots \otimes \rho_{B}$, where $\rho_{B}=|B\rangle\langle B|$ describes an initially Bell-entangled pair. The density matrix $\rho_{i}$ describes the initial conditions from which the rest of the quasiclassical realm emerges. The full final state, similarly, is $\rho_{f} \otimes \rho^{\prime} \otimes \cdots \otimes \rho^{\prime}$, where $\rho^{\prime}=I_{L} \otimes|\uparrow\rangle_{R}\left\langle\left.\uparrow\right|_{R}\right.$, and $I_{L}$ is the identity operator on the left hand particle spin. In both cases, the other degrees of freedom describing the initial states have been omitted. The precise choice of the density matrix $\rho_{f}$, which describes the final state of the rest of the quasiclassical realm, is unimportant.

The experimentalists exchange all their data via classical signals, and search for correlations in those data. Familiar with ABL quantum theory, they eventually correctly conclude that the pairs they observe are pre- and post-selected to arrive in the state $\rho_{B}$ and to reach the final state $\rho^{\prime}$.

As they realise, these conditions mean that the outcome probabilities for the experimenters on the left depend on the axes chosen by the experimenters on the right. For instance, if the experimenters on the right choose the $z$ axis, the post-selection implies that they will, with probability one, obtain the eigenvalue $+\frac{1}{2}$. If the experimenters on the left also choose the $z$ axis for the corresponding particle, they will also, with probability one, obtain the eigenvalue $-\frac{1}{2}$. On the other hand, if the experimenters on the right choose the $x$ axis, they will have probability $\frac{1}{2}$ of finding their particle in either of the possible spin eigenstates. The left hand experimenters, if they still choose the $z$ axis, will then also have probability $\frac{1}{2}$ of finding the corresponding particle in either eigenstate.

The experimenters now use classical signals to agree a superluminal signalling protocol for future use. For the right hand experimenters to send a 0 , they choose the $z$ axis for their observations on an agreed sequence of $m$ particles. To send a 1 , they choose the $x$ axis for the same sequence of particles. The left hand experimenters always choose the $z$ axis for their observations. If they obtain the eigenvalue $-\frac{1}{2}$ for each of the $m$ particles, they record a 0 ; if not, they record a 1 .

This is clearly a probabilistic (and not optimally efficient) protocol: the probability, $2^{-m}$, of misreading a 1 as a 0 is small for large $m$, but cannot be made to vanish. Nonetheless, if the spacelike separation is large compared with the interval between the arrival of successive 
particles, the protocol allows long messages to be sent superluminally and very reliably. In information theoretic terms, 8 the information transmitted superluminally is non-zero and can be made arbitrarily close to one bit by taking $m$ large. Standard causality is thus violated.

This signalling protocol will work only under the specified conditions. Actions of the experimenters or later observers could frustrate it. If the particles re-interact with the quasiclassical world after the first measurement, the outcome probabilities will be altered, and the signalling will not necessarily work.

There is no contradiction here, however. The assumptions made in the example correspond to a possible cosmology. If, according to the initial and final conditions of that cosmology, the experimenters are bound - in all histories of the set $\mathcal{S}$ - to carry out precisely one observation on each particle in each pair, and to allow the particles to propagate unhindered, then the protocol will work. If these assumptions hold true in some histories of the set, the protocol will work if the realised history is one of those, and will not necessarily work otherwise. There is no reason why it should be true, in a general cosmology, that the probability of the assumptions being satisfied is large, or even non-zero. However, we may (and do) consider some realised history in some particular cosmology in which they do, in fact, hold.

Whatever the details of the cosmology, any timeneutral theory of GMH type, together with the specification of some consistent quasiclassical set, will unambiguously assign probabilities to possible histories in a logically consistent way. Thus, although the theories allow superluminal signalling, there is no possibility of setting up a paradoxical causal loop in a way which leads to an internal contradiction: some internally consistent outcome will be realised. In standard superluminal signalling paradoxes, a signaller can send signals into his own past light cone, and one can imagine things being arranged so that the subsequent transmission of the signals then becomes impossible - by, for example, killing the signallers' grandfather as a child. [9] Such paradoxes break down here because the probabilistic predictions in a time-neutral model are context-dependent. Superluminal signalling devices can have efficiency arbitrarily close to one in isolation, but if a paradoxical causal loop is set up involving such devices, at least one device will fail to function - not through any mysterious intervention or because of any ad hoc postulate, but as a result of the standard probability rules of the consistent histories formalism.

Nor is there any violation of Lorentz invariance in any standard sense: the theory itself picks out no preferred frame, and its predictions can equally well be calculated in any frame.

\section{CONCLUSIONS}

We have seen that GMH time-neutral cosmologies can allow superluminal signalling, given knowledge of a detectable regularity in the final state - knowledge which can be empirically obtained by observers in those cosmologies.

The example given is admittedly contrived. However, the possibility of superluminal signalling in these cosmologies is more general. Any final state with interesting regularities is liable to allow superluminal signalling. For the above argument to go through, all that is required is that, at some point in a realised history, ensembles of entangled pairs of quantum subsystems can be produced in which at least one member of the pair, if left to propagate freely, would, as a result of post-selection, have a propensity to end up in some particular one of the entangled eigenstates.

For example, the time-neutral cosmologies GMH discuss involve the formation of large-scale structure at both ends of the universe. It is, admittedly, hard to prove any rigorous result about the properties of such cosmologies. However, if they can actually be realised, it seems clear that, from the perspective of observers nearer (what they regard as) the initial state, there should be a propensity for matter eventually to propagate into the stars and galaxies formed in the time-reversed sense at the other end of the cosmology, in a way which eventually must result in a detectable deviation from the predictions of the time asymmetric quantum theory defined by the initial state alone. It should then be possible to use this propensity to construct superluminal signalling devices of the type described above, by using pairs of particles entangled in position space.

Paradox is avoided as the probability that any signal is successfully transmitted is context-dependent. Signalling devices, however reliable when operated in isolation, will generally become unreliable if any paradoxical loop is attempted. Though superluminal signalling is usually seen as a fatal flaw, it causes no inconsistency in time-neutral cosmologies.

Of course, there are independent reasons to approach GMH time-neutral cosmologies with scepticism. The hypothesis that initial causes suffice seems very well established. Also, the evidence appears to lean against a closed universe, and a closed universe is the natural cosmological setting for time-neutral models,

Still, it seems clear that the reason why standard quantum theory respects Minkowski space causality — in the sense that it does not allow superluminal signalling is not quite as usually suggested. It is not absolutely necessary to forbid superluminal signalling in order to maintain the internal consistency of a relativistic quantum theory. The impossibility of superluminal signalling is, rather, ultimately a hypothesis, contingent on the fact 
that naturally occurring ensembles can be identified on the basis of pre-selection alone.

Price has recently suggested [10] that backward and forward causation could in principle simultaneously be present in a well-defined physical theory. It follows from the discussion above that this is indeed a property of suitable time-neutral cosmological theories. To explain the sense in which this is so, we need first to say something about the language of causality in standard time asymmetric theories. ${ }^{4}$

As mentioned above, according to essentially all current physical theories, our actions are in principle probabilistically predictable from the boundary conditions. Nonetheless, it is generally regarded as useful to employ the language of causality in describing of our actions to say, for example, that we are able to causally influence the future, but not the past. Various justifications are given. It is sometimes intended that causes and effects are to be distinguished by the asymmetry of their correlations. Many independent future effects are generally correlated with, and can be traced to, a single cause. If, for example, we send an ordinary radio signal, it may be received and acted upon by many people in the future. Causal statements about the consequences of our own actions can also be meant simply as statements about our own perspective on the world. It appears to us that we have a free choice to send the signal or not, and that the events which follow from our sending the signal are contingent on and caused by our exercising the choice to send it.

In either of these conventional senses, when timeneutral cosmological theories allow superluminal signalling, they generally allow bidirectional causation. ${ }^{5}$ Agents in those theories who, from their perspective, have evolved to causally influence the future, can also causally influence the past, for example by setting up two or more superluminal signalling devices connected in such a way as to send signals into their past light cone - when, that is, the boundary conditions allow such constructions, and some boundary conditions certainly do. Again, though, the past can never be influenced in a way which will lead to contradictions - it is always impossible, for example, for agents to undo their present observations by acting on the past.

\footnotetext{
${ }^{4}$ For a detailed discussion of causality the reader is enthusiastically referred to Ref. 10]. The view sketched in the following brief comments is indebted to, but not necessarily endorsed by, and certainly not an adequate summary of, Price's discussion.

${ }^{5}$ More generally, the claim is that there is no sensible way of maintaining the language of causation, with respect to the actions of agents like ourselves, in GMH time-neutral theories, without admitting the possibility of bidirectional causation.
}

Finally, the fact that time-neutral theories allow superluminal signalling may have interesting implications for discussions of the properties of other non-standard variants of quantum theory. It is often suggested that nonrelativistic theories which allow superluminal signalling - for example, non-linear generalisations of quantum mechanics - cannot possibly have a consistent relativistic extension. While this may indeed be true in specific cases of interest, some further argument is clearly needed: the demonstration of superluminal signalling is not sufficient per se.

Of course, one can always argue that theories which allow superluminal signalling should be rejected on grounds of aesthetics or by Ockham's razor. The discussion above, which is solely concerned with the logical relations among physical hypotheses, provides no counter-argument here.

Acknowledgements I am grateful to colleagues at York University, Cambridge University, King's College, London, and Oxford University for helpful comments in recent seminars on this topic, and to the Royal Society for financial support.

[1] Y. Aharonov, P. Bergmann and J. Lebowitz, Phys. Rev. B 134 (1964) 1410

[2] M. Gell-Mann and J.B. Hartle in Complexity, Entropy, and the Physics of Information, SFI Studies in the Sciences of Complexity, Vol. VIII, W. Zurek (ed.), Addison Wesley, Reading (1990).

[3] M. Gell-Mann and J.B. Hartle, Phys. Rev. D 47 (1993) 3345

[4] F. Dowker and A. Kent, J. Stat. Phys. 82 (1996) 1575

[5] F. Dowker and A. Kent, Phys. Rev. Lett. 75 (1995) 3038

[6] M. Gell-Mann and J.B. Hartle in Proceedings of the NATO Workshop on the Physical Origins of Time Asymmetry, Mazagón, Spain, September 30-October 4, 1991, J. Halliwell, J. Pérez-Mercader, and W. Zurek (eds.), Cambridge University Press, Cambridge (1994).

[7] D. Craig, Ann. Phys. 251 (1996) 384

[8] C. Shannon and W. Weaver, The Mathematical Theory of Communication, University of Illinois Press, Urbana, 
IL, (1949).

[9] See e.g. W. Rindler, Essential Relativity, Springer-Verlag, New York (1977), p.36.
[10] H. Price, Time's Arrow and Archimedes' Point: New Directions for the Physics of Time, Oxford University Press, Oxford (1996). 